\title{
The highly selective Bruton tyrosine kinase inhibitor acalabrutinib leaves macrophage phagocytosis intact
}

Treatment of chronic lymphocytic leukemia (CLL), has been transformed by the Bruton tyrosine kinase inhibitor (BTKi) ibrutinib (IBR). Although BTKi treatment mobilizes CLL cells from lymphoid organs into the circulation, ${ }^{1}$ where they are highly susceptible to clearance by antiCD20 monoclonal antibodies (mAb), ${ }^{2}$ randomized clinical trials showed no advantage of adding the anti-CD20 mAb rituximab to IBR for treatment of CLL. ${ }^{2,3}$ IBR inhibition of antibody-dependent cellular phagocytosis (ADCP) by splenic and liver macrophages, the principal mechanism of action of anti-CD20 mAb, ${ }^{4}$ might explain this result. Macrophage ADCP requires immunoglobulin Fc $\gamma$ receptors (FcR) binding to $\mathrm{mAb}$-opsonized target cells. Because FcR signaling may involve BTK, ${ }^{5}$ BTKi could prevent the therapeutic effects of anti-CD20 mAb. Indeed, studies have found that IBR decreases ADCP in vitro. ${ }^{6,7}$ In contrast, the highly selective BTKi acalabrutinib (ACALA) with fewer offtargets than IBR $(\sim 5 \text { vs. }>20)^{8,9}$ does not significantly decrease in vitro $A D C P .6,7$ We hypothesized that suppression of ADCP by IBR is mediated by off-target inhibition unique to IBR. (Figure 1A). ACALA also causes less lymphocytosis than IBR, ${ }^{10}$ leading to the hypothesis that IBR, but not ACALA, inhibits phagocytosis of apoptotic cells (efferocytosis) ${ }^{11}$ by a more generalizable off-target effect on phagocytosis. In order to test these hypotheses, we measured human monocyte-derived macrophage (hMDM) phagocytic engulfment and processing of CLL cell targets in vitro. ${ }^{7,12}$

Human specimen collection and usage was conducted with written informed consent after approval of the University of Rochester Research Subjects Review Board according to the ethical guidelines of the Declaration of Helsinki. We examined the effect of IBR or ACALA on hMDM ADCP of anti-CD20 mAb opsonized CLL cells in real time using live cell time-lapse video imaging that visualizes phagocytic engulfments as "voids" in Cell Tracker Deep Red (CTDR, Thermo Fisher Scientific)-labeled hMDM (Online Supplementary Figure S1). ${ }^{12}$ Images of $10 \mu \mathrm{g} / \mathrm{mL}$ rituximab-mediated ADCP of CLL cells by CTDR-labeled hMDM (20:1 CLL:hMDM ratio) with or without IBR or ACALA were captured every 4 minutes ( $\mathrm{min}$ ) over 2.8 hours $(h)$ in 18 replicate experiments. In order to enable detection of off-target effects, the range of BTKi concentrations (serial dilutions from 0.41-100 $\mu \mathrm{M}$ ) was chosen to encompass and span above the mean clinical peak free drug concentration $\left(C_{\max }\right.$ is $\sim 0.5 \mu \mathrm{M}$ and $\sim 1.2 \mu \mathrm{M}$ for IBR and ACALA, respectively $\left.{ }^{1,10}\right)$. Imaging showed a visually apparent reduction in ADCP after 60 min with IBR but not ACALA (Figure 1B). Further examples of ADCP time-lapse images and videos of IBR or ACALA treatment with combined CTDR and Phase channels or CTDR channel alone are available in data sharing statement (Supplementary Figure DS1; Supplementary Videos DS5 to 8). ADCP engulfment events were quantified by void index and graphically displayed as previously described..$^{12,13}$ Rituximab alone caused the ADCP void index to rapidly increase and approach maximum by $1 \mathrm{~h}$, which is the initial engorgement phase observed during ADCP kinetics ( $0 \mu \mathrm{M}$, Figure 1C; Online Supplementary Figure $S 2 A){ }^{13}$ IBR exhibits a concentrationdependent inhibition of ADCP from $0.41-100 \mu \mathrm{M}$ during this phase, while ACALA only inhibited at the highest $100 \mu \mathrm{M}$ concentration (Figure 1C; Online Supplementary Figure $S 2 A)$. In order to analyze this kinetic inhibition, ADCP was summarized as area under the curve (AUC) in the first hour of treatment. Serial dilutions of each drug compared to untreated showed that IBR significantly inhibited ADCP at all measured concentrations $(0.41-100 \mu \mathrm{M}, P<0.05$; Figure 1D). In contrast, ACALA did not significantly inhibit $A D C P$ at concentrations $<100 \mu \mathrm{M}(P>0.05$; Figure 1D). Moreover, comparison of relative ACALA versus IBR inhibition as a ratio showed significantly higher inhibition by IBR at all measured drug concentrations (0.41-100 $\mu \mathrm{M}$, $P<0.01$; Figure $1 \mathrm{E}$ ), which may be slightly overestimated due to an unexpected increase in ADCP at low ACALA concentrations (Figure 1C). These results confirm and extend previous studies using indirect or semi-quantitative single time point observations that suggest that IBR but not ACALA inhibits ADCP. ${ }^{6,714}$ Because ACALA is a more selective inhibitor of BTK, these data imply that BTK inhibition is not responsible for the decreased ADCP measured in hMDM treated with IBR. Inhibition of ADCP by IBR is likely the result of IBR-specific off-target effects (Figure 1A). In order to determine if IBR specific off-target effects broadly alter phagocytosis, we studied the effect of IBR and ACALA on hMDM efferocytosis, an antibody-independent form of phagocytosis that does not involve BTK (Figure $1 A) \cdot{ }^{11}$ Initial measurements of efferocytosis were done by flow cytometry at a single time point. Treatment of hMDM with IBR or ACALA at concentrations ranging from 1.25-10 $\mu \mathrm{M}$ showed no significant effect of either drug on the percentage of hMDM efferocytosis ( $P>0.05$; Figure $2 A$ ). In order to determine if either drug had any effects on the kinetics of efferocytosis, we used the live cell time-lapse video imaging approach using pHrodo Red-labeled apoptotic CLL cells as targets for phagocytosis by CTDR-labeled hMDM (20:1 CLL:hMDM ratio) either untreated or with IBR or ACALA (2-fold serial dilutions from 10-1.25 $\mu \mathrm{M}$ ). Images of duplicate or triplicate wells for each drug con- 

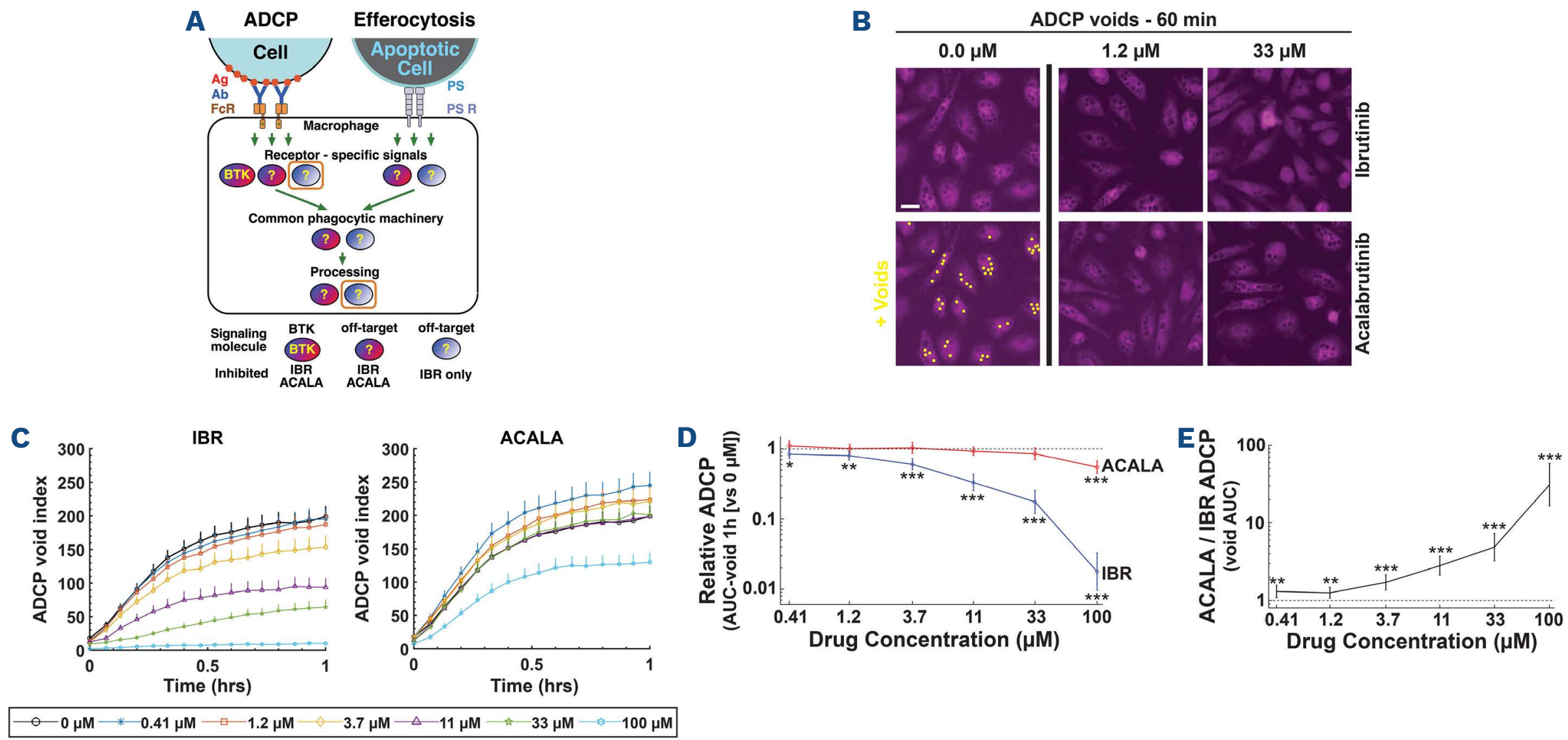

Figure 1. Bruton tyrosine kinase inhibitor effects on phagocytosis and antibody-dependent cellular phagocytosis measurements. (A) Schematic of ibrutinib (IBR) or acalabrutinib (ACALA) inhibition of phagocytosis (antibody-dependent cellular phagocytosis $[A D C P]$ or efferocytosis) shows ADCP (left) proceeding via target cell antigen (Ag) binding to antibody (Ab) with the Fc portion binding to the Fc receptor (FCR) on the macrophage, resulting in FcR signaling via BTK and/or other molecules that may be offtargets inhibited by IBR and/or ACALA. Efferocytosis (right) proceeds via phosphatidylserine (PS) exposure on apoptotic cell surfaces that are bound by macrophage PS receptors (PS R), resulting in signaling via potential off-target molecules. Both pathways lead to common phagocytic machinery and subsequent phagolysosomal processing via potential off-target molecules. IBR offtarget inhibition affects FCR-mediated signaling and downstream phagolysosomal processing (gold rectangles). (B) Representative time-lapse video images ( $8 \%$ subsection of full field of view of the CTDR channel) of anti-CD20 mAb-mediated ADCP of chronic lymphocytic leukemia (CLL) cells by human monocyte-derived macrophage (hMDM) visually show phagocytic engulfment voids at 60 minutes (min) with 0.0, 1.2 and $33 \mu \mathrm{M}$ of IBR (top) or ACALA (bottom), but with less voids at $33 \mu \mathrm{M}$ IBR. Duplicate $0.0 \mu \mathrm{M}$ image (+ Voids) highlights voids with yellow spots. Scale bar $=25 \mu \mathrm{M}$. Images of all drug concentrations are available in the data sharing statement (Supplementary Figure DS1). (C) Phagocytic engulfments were measured by phagocytic void index (means $(n=18)+$ standard error) every 4 min and plotted for IBR (left) and ACALA (right) at indicated drug concentrations for the first 1.0

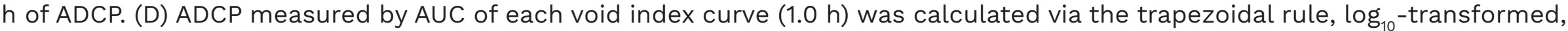
and modeled as a function of drug concentration, dose and their interaction. All hypothesis tests were performed at the 2-sided 0.05 level using SAS 9.4 (SAS Institute, Inc. Cary, NC, USA). Means and associated 95\% confidence intervals (CI) of mixed modelbased contrasts of each concentration with untreated $(0 \mu \mathrm{M})$ shows relative ADCP $(* P<0.05, * * P<0.01, * * * P<0.001)$. Dotted line marks no change relative to $0 \mu \mathrm{M}$ with values below indicating inhibition. (E) The difference between ACALA and IBR was assessed by mixed model-based contrasts with means and associated $95 \% \mathrm{Cl}$ shown for each concentration ( $* * P<0.01, * * * P<0.001)$. Dotted line marks drug equivalence with values above indicating more inhibition of ADCP by IBR than ACALA.

centration were collected every 4 min over $2.8 \mathrm{~h}$ in seven replicate experiments. Efferocytosis was visible with no apparent differences after drug treatment $(2.5 \mu \mathrm{M}$ or 10 $\mu \mathrm{M}, 60 \mathrm{~min}$; Figure 2B). Further examples of efferocytosis time-lapse images and videos with IBR or ACALA treatment are available in data sharing information (Supplementary Figure DS3; Supplementary Videos DS9, DS10, $D S 13$ and DS14). Quantitation of engulfments by void index indicate that IBR and ACALA did not alter engulfment kinetics across all drug concentrations (1.25-10 $\mu \mathrm{M}$; Figure 2B; Online Supplementary Figure S2B). Quantitation by $A U C$ of the void index plot over the first $1 \mathrm{~h}$ showed no significant difference between untreated and treatment with any concentration of either drug ( $P>0.05$; Figure 2D). Because IBR or ACALA did not inhibit efferocytosis, these data suggest that BTK and off-target molecules inhibited by IBR are not involved in initial signaling mediated by the receptors for apoptotic cells in efferocytosis or common downstream phagocytosis pathway signaling (Figure 1A). This lack of effect of IBR and ACALA on apoptotic cell phagocytosis in vitro suggests that inhibition of efferocytosis does not cause the greater lymphocytosis seen clinically with IBR treatment, ${ }^{1,10}$ and provided an opportunity to study the effects of IBR or ACALA on downstream phagolysosomal processing.

Phagosomes with internalized cargo transition to acidified phagolysosomes for processing. ${ }^{2}$ The kinetics of this process can be measured by target cells labeled with $\mathrm{pH}$-sensitive dyes, such as pHrodo Red, which increase in intensity with decreasing $\mathrm{pH}^{12}$ Efferocytosis by hMDM 
A

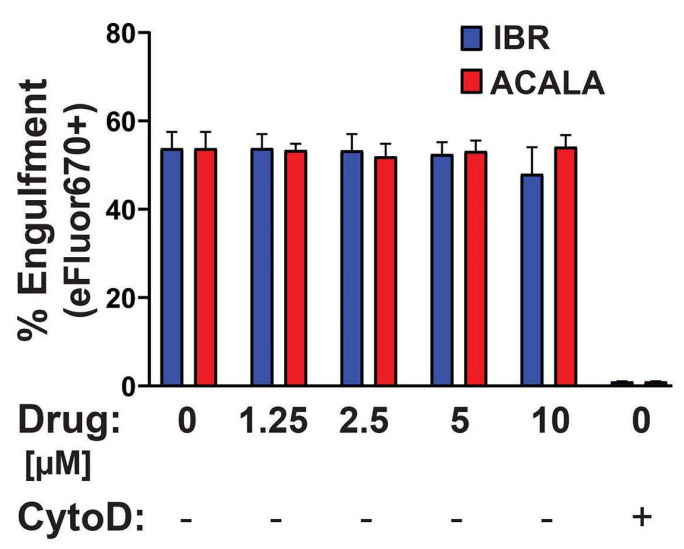

B

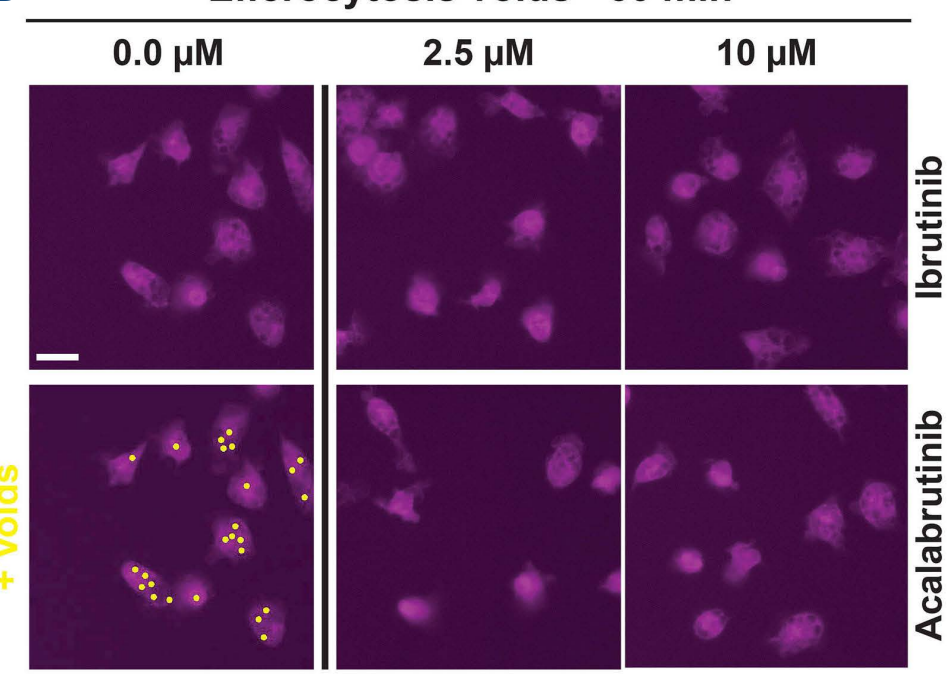

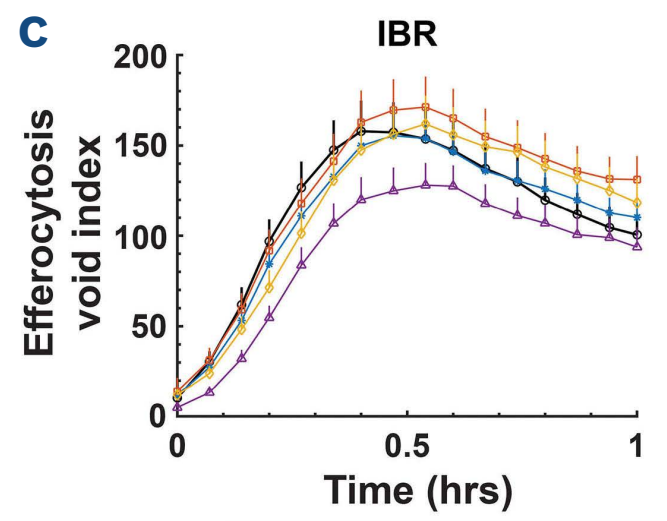

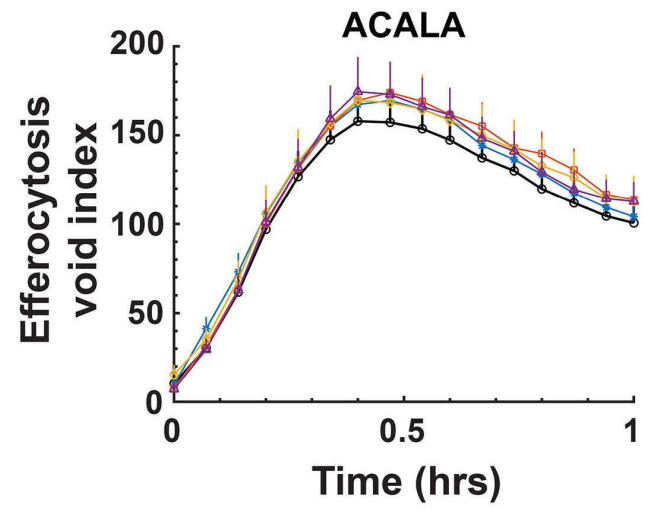

Time (hrs)

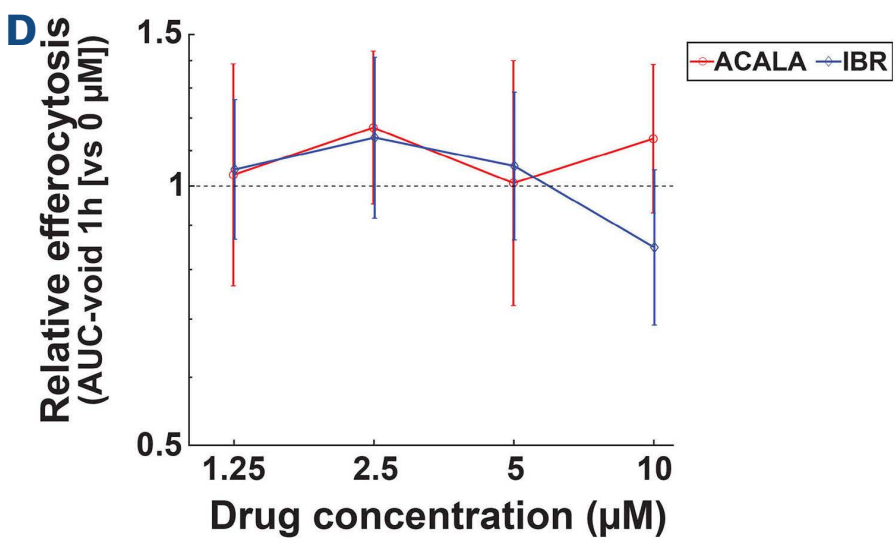

Figure 2. BTK inhibitors have little effect on efferocytosis. (A) Percent human monocyte-derived macrophage (hMDM) that engulfed eFluor670+ apoptotic chronic lymphocytic leukemia (CLL) cells after treatment with 0-10 $\mu \mathrm{M}$ drug is shown after 1 hour (h) by flow cytometry with no significant change for ibrutinib (IBR) or acalabrutinib (ACALA) (mean [n=4] + standard error). Cytocholasin D (CytoD) prevents phagocytosis and serves as a control. (B) Representative time-lapse video images ( 8\% subsection of full field of view of the CTDR channel) of efferocytosis show no visual decrease in phagocytic engulfment voids following treatment $(0.0,2.5,10 \mu \mathrm{M})$ with IBR (top) or ACALA (bottom). Duplicate $0.0 \mu \mathrm{M}$ image (+ Voids) highlights voids with yellow spots. Scale bar $=25 \mu \mathrm{M}$. Images of all drug concentrations are available in data sharing statement (Supplementary Figure DS3). (C) Efferocytosis phagocytic engulfments were measured by phagocytic void index (means [ $n=7$ ] + standard error) every 4 minutes (min) and plotted for IBR (left) and ACALA (right) at indicated drug concentrations for the first $1.0 \mathrm{~h}$ of efferocytosis. (D) Efferocytosis, as measured by the area under the curve (AUC) of the $1^{\text {st }} \mathrm{h}$ of each void index curve, was calculated and modeled as in Figure 1D. Means and associated 95\% confidence intervals (CI) of mixed model-based contrasts of each concentration with untreated are shown. Dotted line indicates no change relative to $0 \mu \mathrm{M}$. There was insufficient evidence of any difference from untreated $(P>0.05)$.

of pHrodo Red-labeled apoptotic CLL cells produces a readily visualized increase in dye intensity after $2 \mathrm{~h}$ that colocalizes with phagocytic voids (Figure $3 A$ ). Effects of IBR or ACALA drug treatment on this change in dye intensity were not easily visualized on inspection as illustrated in representative $2.5 \mu \mathrm{M}$ and $10 \mu \mathrm{M}$ images (Figure $3 A)$. Further examples of IBR or ACALA treated phagolysosomal processing time-lapse images and videos are available in the data sharing statement (Supplementary Figure DS4; Supplementary Videos DS11, DS12, DS15 and $D S 16)$. In order to quantitate pHrodo Red dye intensity levels normalized to macrophage number, the dye intensity index was calculated and plotted over time (Figure 3B; Online Supplementary Figure S2C). ${ }^{12,13}$ The kinetics of the dye intensity index demonstrated the expected delay relative to the void index (Figure 2C; Online Supplemen- tary Figure S2B), because phagolysosomal processing occurs after phagocytic engulfments. ${ }^{2}$ Higher concentrations of IBR but not ACALA exhibited a decrease in the dye index relative to untreated (Figure 3B; Online Supplementary Figure S2C). For phagolysosomal processing, the AUC was calculated for $2 \mathrm{~h}$ and the drug concentration curves were analyzed. Compared to untreated, there was a progressive decrease in phagolysosomal processing with increasing IBR but not ACALA concentrations, which was significant at $10 \mu \mathrm{M}(P=0.008$; Figure $3 C)$. When comparing the effects of ACALA versus IBR, there was a progressive increase in this ratio with increased drug concentration that was significant at $10 \mu \mathrm{M}$ $(P=0.0002$; Figure 3D). Since ACALA had no effect on phagolysosomal processing, these results suggest that BTK signaling is not essential for this process and that 
A

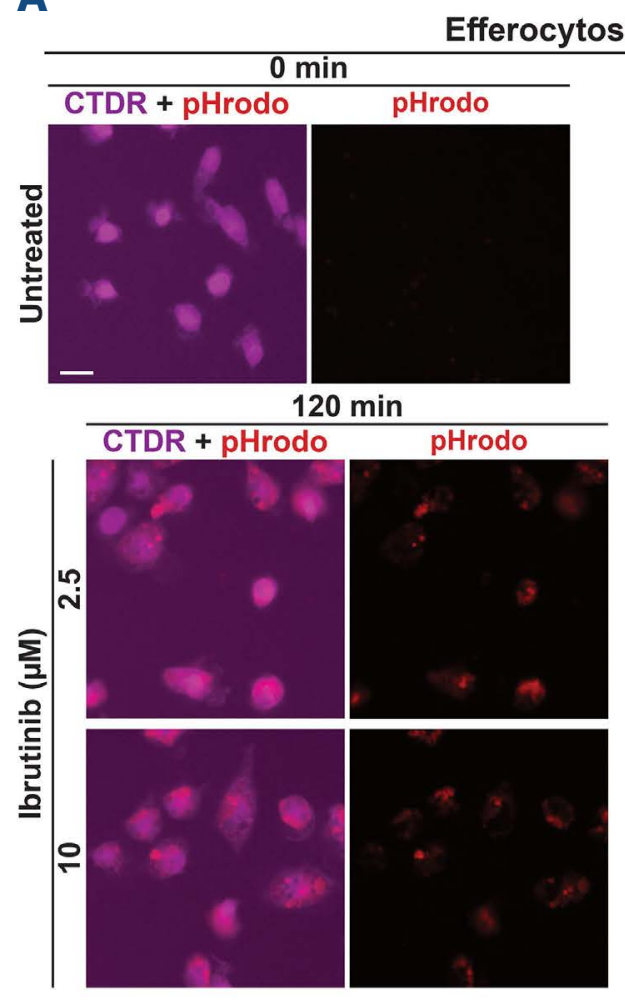

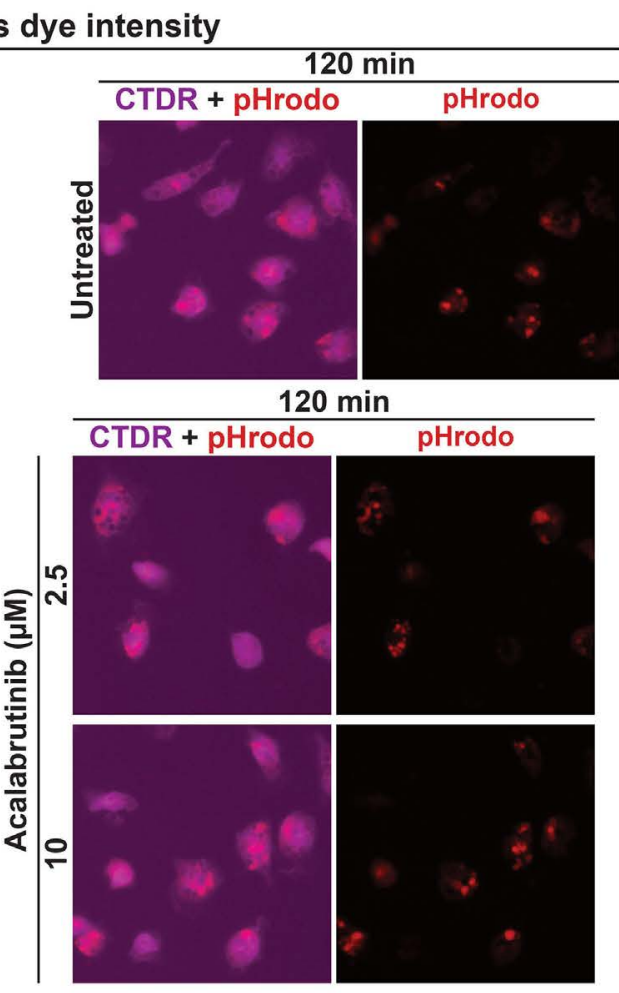

B

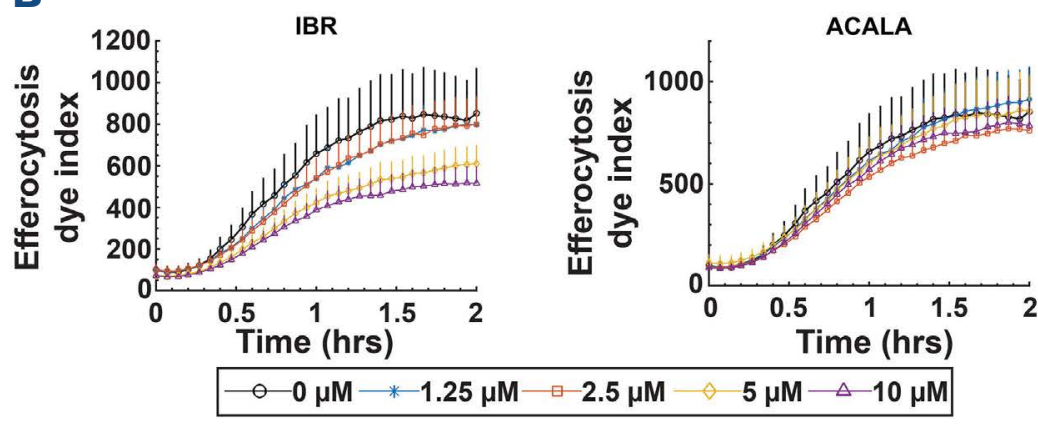

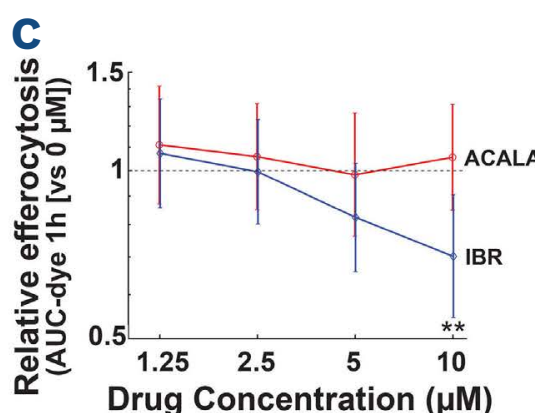

D

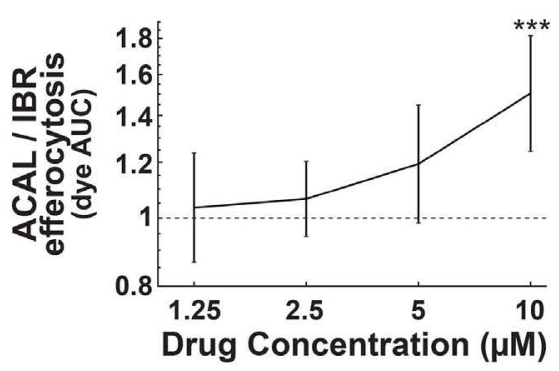

Figure 3. Phagolysosomal processing is inhibited by ibrutinib, but not by acalabrutinib. Live cell time-lapse video imaging of efferocytosis was collected as in Figure 2. (A) In order to illustrate the appearance of pHrodo Red dye intensity, time-lapse video images of the combined Cell Tracker Deep Red (CTDR) and pHrodo Red channels (CTDR + pHrodo) or pHrodo Red channel alone (pHrodo) from the representative well and drug concentrations in Figure 2 are shown for untreated (0 and 120 minutes [min]) and ibrutinib (IBR) (left) or acalabrutinib (ACALA) (right) treatment (120 min). Scale bar $=25 \mu \mathrm{M}$. Images of all drug concentrations are available in data sharing statement (Supplementary Figure DS4). (B) Live cell time-lapse high-content microscopy imaging collected from experiments shown in Figure 2 was measured by dye intensity index to assess phagolysosomal processing. Efferocytosis dye intensity index time course over 2.0 hours is shown for IBR (left) and ACALA (right) with drug concentrations indicated. The mean of 6 experiments for each time-point measured in duplicate or triplicate is shown with positive standard error bars. (C) Phagolysosomal processing during efferocytosis as measured by area under the curve (AUC) of the first 2 hours of each dye intensity curve, was modeled as a function of drug concentration, dose and their interaction. Means and associated $95 \%$ confidence intervals $(\mathrm{Cl})$ of mixed model-based contrasts of each concentration with untreated are shown. Dotted line indicates no change relative to $0 \mu \mathrm{M}$. Significant difference from untreated was only observed at $10 \mu \mathrm{M}$ IBR concentration ( $* \star P<0.01$ ). (D) Mixed model-based contrasts were built to assess the difference between drugs at every concentration. Means and associated $95 \% \mathrm{Cl}$ are shown. Dotted line indicates drug equivalence. Values above dotted line indicate more inhibition of efferocytosis by ibrutinib than acalabrutinib. Significant difference between drugs was only seen at $10 \mu \mathrm{M}$ drug concentration ( $* \star * P<0.001)$.

IBR effects are mediated by off-target inhibition (Figure 1A). IBR obstruction of phagolysosomal processing could result in delay of upstream phagocytosis. Future studies will be needed to study IBR-inhibited off-target(s) in phagocytosis.

These data show that short-term highly selective BTK inhibition in vitro by ACALA does not alter macrophage functions of $\mathrm{mAb}$ mediated ADCP, antibody-independent efferocytosis, or phagolysosomal processing. In contrast, IBR significantly inhibited ADCP over a wide range of drug concentrations $(0.41-100 \mu \mathrm{M})$. Thus, inhibition of ADCP, the principal mechanism of therapeutic anti-CD20 mAb efficacy, ${ }^{4}$ could explain the lack of clinical benefit for the addition of anti-CD20 mAb rituximab to IBR for treatment of CLL. ${ }^{2,3}$ These data suggest that addition of antiCD20 mAb to a more selective BTKi would be a preferable choice in the treatment of patients with Bcell malignancies such as CLL. Furthermore, newer highly selective reversible $\mathrm{BTKi}$, would be of interest to assess in combination with anti-CD20 mAb therapy. ${ }^{15}$

\section{Authors}

Jonathan J. Pinney, ${ }^{1,2}$ Sara K. Blick-Nitko, ${ }^{3}$ Andrea M. Baran, ${ }^{4}$ Derick R. Peterson, ${ }^{4}$ Hannah E. Whitehead ${ }^{1,2}$ Raquel Izumi, ${ }^{5}$ Veerendra Munugalavadla, ${ }^{6}$ Karl R. VanDerMeid, ${ }^{7,8}$ Paul M. Barr, ${ }^{7,8}$ Clive S. Zent, ${ }^{7,8}$ Michael R. Elliottt ${ }^{1,2,9}$ and Charles C. Chu ${ }^{7,8}$

${ }^{1}$ Center for Vaccine Biology and Immunology, and ${ }^{2}$ Department of Microbiology and Immunology, University of Rochester, Rochester, NY; ${ }^{3}$ Department of Pathology and Laboratory Medicine, University of Rochester, Rochester, NY; ${ }^{4}$ Department of Biostatistics and Computational Biology, University of Rochester Medical Center, Rochester, NY; ${ }^{5}$ Acerta Pharma, a member of the AstraZeneca Group, South San Francisco, CA; ${ }^{6}$ AstraZeneca, South San Francisco, CA; ${ }^{7}$ Department of Medicine, University of Rochester Medical Center, Rochester, NY, ${ }^{8}$ Wilmot Cancer Institute, University of Rochester Medical Center, Rochester, NY and ${ }^{9}$ Center for Cell Clearance and the Department of Microbiology, Immunology, and Cancer Biology, University of Virginia, Charlottesville, VA, USA. 


\section{LETTER TO THE EDITOR}

\section{Correspondence:}

Charles C. Chu - charles_chu@urmc.rochester.edu

Michael R. Elliott - mre4n@virginia.edu

https://doi.org/10.3324/haematol.2021.279560

Received: July 7, 2021.

Accepted: February 9, 2022.

Prepublished: February 17, 2022.

\section{Disclosures}

AMB received research funding from Acerta Pharma / AstraZeneca; RI has equity ownership in Acerta Pharma and AstraZeneca; was employed by Acerta Pharma (during time of study); and holds patents from intellectual property for Acerta Pharma including patents surrounding acalabrutinib; VM is employed by Acerta Pharma (a member of the AstraZeneca group) and has equity ownership in AstraZeneca and Gilead Sciences; KRV received research funding from Acerta Pharma / AstraZeneca; PMB consults for Pharmacyclics LLC / AbbVie, AbbVie, Genentech, Gilead, Merck, Seattle Genetics, Verastem, AstraZeneca, Celgene, Morphosys, TG Therapeutics, and Janssen; and receives research funding from Pharmacyclics LLC / AbbVie, TG Therapeutics, and AstraZeneca; CSZ received research funding from Acerta Pharma / AstraZeneca, Mentrik Biotech, and TG Therapeutics; MRE received research funding from Acerta Pharma / AstraZeneca; CCC has equity ownership in Pfizer and received research funding from Acerta Pharma / AstraZeneca and TG Therapeutics. All other authors report no conflicts of interest.

\section{Contributions}

MRE, CSZ, JJP, and CCC planned the study strategy; JJP, SKB-N, and CCC performed experiments; AMB and DRP performed statistical analyses; PMB and CSZ provided clinical samples; HEW and KRV provided cells and reagents for experiments; MRE, CSZ, JJP, AMB, DRP and CCC interpreted data; MRE, CSZ, RI, VM, CCC acquired funding, managed collaboration, and reviewed manuscript; MRE, CSZ, JJP, AMB, DRP and CCC wrote the manuscript.

\section{Acknowledgments}

The authors are grateful to members of the Center for Vaccine Biology and Immunology, University of Rochester Medical Center (URMC), Wilmot Cancer Institute (URMC), Department of Pathology and Laboratory Medicine (URMC), and the Center for Cell Clearance, University of Virginia (UVA) for critical feedback on this project and manuscript; Genentech for anti-CD20 mAb; Drs. Ron Taylor and
Margaret Lindorfer (UVA) for critical feedback throughout the project; Deb Gilbertson and Cecile Krejsa for critical discussions on this project; Rachel Pinney for advice on figure design; CLL patients for participation in our research and donation of blood specimens; Sharon Lewinski RN and Tania Orzol NP for specimen collection, and the New York-Penn branch of the American Red Cross for supplying healthy donor leukocytes.

\section{Funding}

This study was supported by research funding from Acerta Pharma LLC to MRE, CCC, PMB, AMB, and CSZ, by grants to MRE from NIH (grant number: Al114554, DK119285), and the University of Rochester Research Award, Wilmot Cancer Institute and to CSZ (University of Rochester Research Award, Wilmot Cancer Institute), and by philanthropic donation from Ms. Elizabeth J. Aaron to CSZ. JJP was supported in part by the University of Rochester Immunology Training T32 Grant from NIH (grant number: Al007285).

\section{Data sharing statement}

Additional data sharing available upon request. In order to further illustrate the phagocytic quantitation method, Supplementary Videos DS1 to 4 are available in support of Online Supplementary Figure S1. In order to complement Figure 1B, representative timelapse video images of ADCP phagocytic engulfment voids for all drug concentrations of IBR or ACALA are available in the Supplementary Figure DS1 and examples of full-length time-lapse videos using $3.7 \mu \mathrm{M}$ concentration of IBR or ACALA with combined CTDR and Phase channels or CTDR channel alone are available in Supplementary Videos DS5 to 8. Preparation of apoptotic CLL cells by venetoclax treatment for efferocytosis assays is available in Supplementary Figure DS2. In order to complement Figure 2B, representative time-lapse video images of efferocytosis phagocytic engulfment voids for all drug concentrations of IBR or ACALA are available in Supplementary Figure DS3 and examples of full-length time-lapse videos using $10 \mu \mathrm{M}$ concentration of IBR or ACALA with combined CTDR and Phase channels or CTDR channel alone are available in Supplementary Videos DS9, DS10, DS13 and DS14. In order to complement Figure $3 \mathrm{~A}$, representative time-lapse video images of efferocytosis changes in phagolysosomal processing dye intensity for all drug concentrations of IBR or ACALA are available in Supplementary Figure DS4 and examples of full-length time-lapse videos using $10 \mu \mathrm{M}$ concentration of IBR or ACALA with combined CTDR and Phase channels or CTDR channel alone are available in Supplementary Videos DS11, DS12, DS15 and DS16.

\section{References}

1. Byrd JC, Furman RR, Coutre SE, et al. Targeting BTK with ibrutinib in relapsed chronic lymphocytic leukemia. N Engl J Med. 2013;369(1):32-42.

2. Burger JA, Keating MJ, Wierda WG, et al. Safety and activity of ibrutinib plus rituximab for patients with high-risk chronic lymphocytic leukaemia: a single-arm, phase 2 study. Lancet Oncol. 2014;15(10):1090-1099.
3. Rogers A, Woyach JA. BTK inhibitors and anti-CD20 monoclonal antibodies for treatment-naive elderly patients with CLL. Ther Adv Hematol. 2020;11:2040620720912990.

4. Grandjean CL, Montalvao F, Celli S, et al. Intravital imaging reveals improved Kupffer cell-mediated phagocytosis as a mode of action of glycoengineered anti-CD20 antibodies. Sci Rep. 2016;6:34382. 


\section{LETTER TO THE EDITOR}

5. Nimmerjahn F, Ravetch JV. Fcgamma receptors as regulators of immune responses. Nat Rev Immunol. 2008;8(1):34-47.

6. Da Roit F, Engelberts PJ, Taylor RP, et al. Ibrutinib interferes with the cell-mediated anti-tumor activities of therapeutic CD20 antibodies: implications for combination therapy. Haematologica. 2015;100(1):77-86.

7. VanDerMeid KR, Elliott MR, Baran AM, Barr PM, Chu CC, Zent CS. Cellular cytotoxicity of next-generation CD20 monoclonal antibodies. Cancer Immunol Res. 2018;6(10):1150-1160.

8. Barf T, Covey T, Izumi R, et al. Acalabrutinib (ACP-196): a covalent Bruton tyrosine kinase inhibitor with a differentiated selectivity and in vivo potency profile. J Pharmacol Exp Ther. 2017;363(2):240-252.

9. Honigberg LA, Smith AM, Sirisawad M, et al. The Bruton tyrosine kinase inhibitor PCl-32765 blocks B-cell activation and is efficacious in models of autoimmune disease and B-cell malignancy. Proc Natl Acad Sci U S A. 2010;107(29):13075-13080.

10. Byrd JC, Harrington B, O'Brien S, et al. Acalabrutinib (ACP-196) in relapsed chronic lymphocytic leukemia. N Engl J Med. 2016;374(4):323-332.

11. Arandjelovic S, Ravichandran KS. Phagocytosis of apoptotic cells in homeostasis. Nat Immunol. 2015;16(9):907-917.

12. Chu CC, Pinney JJ, Whitehead HE, et al. High-resolution quantification of discrete phagocytic events by live cell timelapse high-content microscopy imaging. J Cell Sci. 2020;133(5):jcs237883.

13. Pinney JJ, Rivera-Escalera F, Chu CC, et al. Macrophage hypophagia as a mechanism of innate immune exhaustion in mAb-induced cell clearance. Blood. 2020;136(18):2065-2079.

14. Golay J, Ubiali G, Introna M. The specific Bruton tyrosine kinase inhibitor acalabrutinib (ACP-196) shows favorable in vitro activity against chronic lymphocytic leukemia B cells with CD20 antibodies. Haematologica. 2017;102(10):e400-e403.

15. Wen T, Wang J, Shi Y, Qian H, Liu P. Inhibitors targeting Bruton's tyrosine kinase in cancers: drug development advances. Leukemia. 2021;35(2):312-332. 